\title{
Concurrent validation of the tandem walk test as a measure of dynamic walking balance in a healthy population
}

Marc Robertson ${ }^{1 *}$ and Robert Gregory ${ }^{1+}$

*Correspondence: robertsonm1@southernct.edu

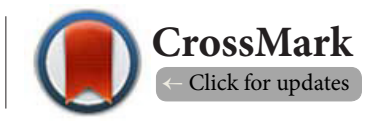

'The author contributed equally to this work.

'Southern Connecticut State University, New Haven, Connecticut, USA.

\begin{abstract}
Background: The purpose of this study was to determine the possible concurrent validity of the tandem walk test as a measure of dynamic walking balance.

Methods: Forty-nine healthy participants, age range 20-75 years (mean age 40.1 \pm 17.1 years), performed the ten step tandem walk test and two clinical tests of dynamic balance using the Biodex Balance System (Biodex Medical Systems, Shirley, NY) dynamic limits of stability protocol and measures of gait variability assessed through gait center of pressure, spatial, and temporal parameters derived from an instrumented treadmill (h/p/cosmos sports \& medical gmbh, Nussdorf-Traunstein, Germany; Zebris FDM-T, Isny im Allgau, Germany).

Results: A moderate-high correlation was demonstrated between the ten step tandem walk test eyes open and measures of gait variability, step width $(\mathrm{r}=0.64 \mathrm{p}<0.05)$ and step time variability $(\mathrm{r}=0.67 \mathrm{p}<0.05)$, age $(r=0.69 \mathrm{p}<0.05)$, and Biodex Balance System dynamic limits of stability $(r=0.81 \mathrm{p}<0.05)$. A moderate relationship was found between the ten step tandem walk test eyes open and measures of gait center of pressure variability $(\mathrm{r}=0.52 \mathrm{p}<0.05)$. Correlation coefficient values between the ten step tandem walk test eyes open and Biodex Balance System dynamic limits of stability, gait temporal, center of pressure, and spatial parameters demonstrated increased strength when adjusted for age.

Conclusion: The ten step tandem walk test demonstrated a moderate to high relationship with clinical tests of dynamic balance: Biodex Balance System dynamic limits of stability and gait center of pressure, spatial, and time parameters in a healthy population demonstrating concurrent validity as an objective measure of dynamic walking balance in a healthy population between the ages of 20 and 75 years.
\end{abstract}

Keywords: Postural balance, validation studies, gait, tandem walk, tandem walk test

\section{Introduction}

Dynamic walking balance is an elusive construct, yet to be quantitatively identified by a gold standard measurement [1]. Lark \& Pasupuleti [2] referenced any situation that maximally narrowed an individual's base of support (BOS) maximally challenged her/his dynamic balance, therefore,walking tests that serve to maximally narrow an individual's BOS, theoretically, should maximally challenge her/his dynamic walking balance. Many laboratory tests and components of functional performance test batteries, such as the Fullerton Advanced Balance
Scale [3,4], the LIFE pilot study protocol [5], Good Walkers Test [6], and the Established Populations for Epidemiologic Studies of the Elderly (EPIDOS) Prospective Study balance protocol [7] reported to test dynamic walking balance, most frequently used to predict those at risk for falls. These test batteries have been validated specifically for the elderly population which has been identified as a population predisposed to impaired dynamic walking balance and falls [8-10] hence, limiting these tests' capacity towards accurately depicting dynamic walking balance in a healthy, younger population. Measures of gait 
variability (center of pressure (COP) [11-13], temporal [14-18] and spatial [19-22] measures) and balance systems, such as the Biodex Balance System (Biodex Medical Systems, Shirley, NY) have commonly been used in the laboratory setting in an attempt to quantify and qualify dynamic walking balance.

Variability of the COP trajectory upon walking has demonstrated the capacity to discriminate between individuals with stable and unstable gait and has been used as a measure of dynamic walking balance [11-13]. COP variability was sensitive to age related changes in gait patterns between middle aged and young female healthy participants [11], utilized as a composite measure of center of mass (COM) and COP to quantify balance control during gait in fall prone and healthy elderly walkers [12], and successfully used to characterize measures of postural control/dynamic balance under both normal and neurologically impaired gait [13].

Temporal gait parameters such as stride time, swing time, and step time variability demonstrated the ability to discriminate between fallers and non-fallers and were considered representative of dynamic walking balance [14,15]. Swing time variability, independent of gait speed, was found to be a valid measure of gait rhythmicity and walking balance [16]. Stride time variability was correlated $(r=0.89 p<.0001)$ with swing time variability and suggested as an indicator of dynamic walking balance [15]. Low stride time variability equated to safe gait and increased gait stability [17]. Participants with neuropathic gait demonstrated increased step time variability which was associated ( $r=0.62 p=.032)$ with unstable walking [18].

Variability within spatial gait parameters, such as step width, step length, and stride length, has equated an increase in variability to an increased risk of falls in elderly individuals [19-22].

The Biodex Balance System (Biodex Medical Systems, Shirley, NY) (BBS) has been used to quantitatively characterize dynamic balance in a multitude of settings. The Biodex Balance System dynamic limits of stability test (BBS DLOS) was used to discriminate dynamic balance between individuals with rheumatoid arthritis and controls [23], very old male and female nursing home residents [24], and for the quantitative assessment of balance in individuals with spinocerebellar ataxia [25]. Clinical utility of the BBS DLOS was determined through intra-tester, inter-tester [26] and test-retest reliability [27]. The BBS DLOS was compared to the NeuroCom Smart Balance Master system (NeuroCom International, Clackamas, $\mathrm{OR}$ ) to assess the dynamic balance discrimination capacity between the two systems [1].

Accurate quantification of gait stability requires sophisticated instrumentation not available to most clinicians [2]. Laboratory measures of dynamic walking balance demonstrate limited clinical utility due to prohibitive cost and portability limitations. The tandem walk test (TWT) has been historically utilized as a reliable measure of dynamic walking balance since 1938 by Dain who measured dynamic balance in boys between the ages of 13 and 18 via a beam walking test and later utilized and refined by Graybiel \& Fregly in 1965 [28-30].
The TWT has frequently been included in standard neurological examinations to indicate neuro-motor competence and dynamic balance capacity [31-33]. The TWT test has been utilized as the dynamic walking balance component of several validated test batteries regarding fall prediction in the elderly, such as the Fullerton Advanced Balance Scale $[3,4]$, the LIFE pilot study protocol [5], Good Walkers Test [6], and the EPIDOS Prospective Study balance protocol [7]. The TWT has been utilized as a test of dynamic walking balance to determine gait stability and pathological quantification including prediction of falls in the elderly population $[3,9,34-40]$, diagnose ataxia and other vestibular disorders $[\mathbf{2 9 , 4 1 ]}$, test for vertigo [42], tropical ataxic neuropathy [43], traumatic brain injury [40], peripheral neuropathy [44], and functional mobility [45-48]. The TWT is considered a clinically efficacious test of dynamic balance in medical text books $[29,49]$ and for research purposes $[2,50]$. The TWT has been utilized as a measure of dynamic walking balance in both clinical and research settings.

The TWT is a simple, commonly used clinical assessment for the purpose of testing dynamic walking balance in patients and study participants. To date, no studies have served to validate the TWT as a clinically efficacious measure of dynamic walking balance in the general population [44], yet there has been a strong assumption that the TWT is a valid measure of dynamic walking balance due to its propensity to maximally narrow individuals' walking BOS [2].

Therefore, the purpose of this investigation was to determine the concurrent validity of the ten step TWT eyes open (TSTWT EO) [8] as a tool for determining dynamic walking balance in a cross-sectional healthy population between the ages of 20 and 75 years to support the hypothesis that the TSTWT EO demonstrates concurrent validity as a test of dynamic walking balance due to its association with laboratory-based tests of dynamic balance. Concurrent validation of the TSTWT EO was tested through examination of its relationship with several laboratory based measures of balance, including the modified Fall Risk Questionnaire (mFRQ) [51], BBS DLOS, and COP, temporal, and spatial measures of gait variability quantified by a $\mathrm{h} / \mathrm{p} / \operatorname{cosmos}(\mathrm{h} / \mathrm{p} / \operatorname{cosmos}$ sports \& medical gmbh, Nussdorf-Traunstein, Germany; Zebris FDM-T, Isny im Allgau, Germany) instrumented treadmill with a Zebris pressure platform utilizing Noraxon (Noraxon U.S.A. Inc., Scottsdale, Arizona) myopressure software.

\section{Methods}

\section{Participants}

This cross-sectional study was conducted at Southern Connecticut State University (SCSU) in greater New Haven County of Connecticut, USA and was approved by the Southern Connecticut State University Institutional Review Board (IRB \#15-139). Forty-nine healthy participants from New Haven County, Connecticut (USA) between the ages of 20 and 75 years were recruited for voluntary test participation. All 
participants completed written informed consent prior to study participation.

Study participant inclusion criteria were: Participants had to be between the ages of 20-75 years old, walk without an assistive device (cane, walker, crutches), no lower extremity or trunk surgeries within the past year, no acute illness or traumatic injury, no unstable health conditions, and had to be able to walk for greater than or equal to four minutes continuously without help/assistance.

\section{Procedures}

1. Participants were explained the test purpose, methodology, and asked to sign the informed consent document. Upon voluntary acceptance of study participation:

2. Participants completed a modified version of Rubenstein and colleagues' Fall Risk Questionnaire (mFRQ) prior to test protocol initiation [51].

3. Each participant wore a standard rehabilitative gait belt (Briggs Healthcare, Waukegan, IL) for the duration of the test session.

4. Participant demographic data were collected [age (years), gender, height, body mass, and body mass index (BMI) $\left.\left(\mathrm{kg} \cdot \mathrm{m}^{-2}\right)\right]$. Height and weight were assessed using a medical scale (Detecto, Webb City, MO-USA).

\section{Tandem walk test}

A fifteen foot long, one inch wide piece of white training tape was placed along a level concrete floor approximately two feet away from and parallel with a wall. The participant was asked to walk forward along the taped line, placing one foot directly in front of the other, as per the heel of one foot directly in front and in contact with the toes of the contralateral foot. Each participant attempted two trials, two sets each for TSTWT EO then TSTWT eyes closed (EC) per trial. Each participant was asked to tandem walk a maximum of ten steps at a comfortable pace with his/her arms folded across his/ her chest without performing a test error including forward progression stoppage, sidestepping, opening the eyes (if applicable), unfolding arms, not maintaining a parallel foot position with the taped line (when applicable), and/or requiring gait belt assistance to maintain an upright position [29]. Test protocol was demonstrated prior to the participant's attempts. Maximum number of steps achieved before error was recorded.

\section{Gait data (COP, spatial, temporal measures) and gait phase parameters}

$\mathrm{COP}$, in relation to dynamic walking balance, is the focal point of location of the vertical ground reaction force vector relative to thefoot in contact with the supporting surface which elicits a COP trajectory as the foot translates on the supporting surface $[11,13]$. The degree of variability within the COP trajectory has been used to quantify static and dynamic postural control in both normal and pathological populations with increased unilateral and side to side variability of the COP trajectory indicative of reduced dynamic walking balance capacity [11-13]. COP measures [ length of gait line left/right $(\mathrm{mm})$ which described the trajectory of the pressure center, accounted for all trajectory planar deviations, and assessed for individual ground contacts on one side of the body; single support line left/right $(\mathrm{mm})$ quantified as the average length of the unilateral trajectory line which described the trajectory of the pressure center and accounted for all ground contacts; and single support line percent difference which described the single support line side to side differences] collected at $300 \mathrm{~Hz}$, gait spatial measures $(\mathrm{cm})$ [step length coefficient of variation (CV) left/right; step length percent difference; step width; and step width CV], temporal measures (s) [step time $C V$; stride time $C V$; and swing time $C V$ ], and gait phase parameters [double stance percentage, cadence, and gait speed] were obtained from participants while walking on a h/p/cosmos-Zebris Medical instrumented treadmill/force platform ( $\mathrm{h} / \mathrm{p} /$ cosmos sports \& medical gmbh, NussdorfTraunstein, Germany; Zebris FDM-T, Isny im Allgau, Germany) with Noraxon Myopressure analytical software (Noraxon U.S.A. Inc., Scottsdale, Arizona). Participants were asked to walk at a comfortable, self-selected speed for four minutes with data, described above, collected over the last 60 seconds of the trial. Each participant was asked not to talk and to look straight ahead while walking on the treadmill. Treadmill speed was determined by advancing the treadmill speed one kilometer per hour until a self-reported comfortable speed was determined; the four minute protocol was then initiated. The participant walked at his/her self-selected pace for three minutes to allow for treadmill walking acclimation with an additional one minute data collection period. The participant was assisted off of the instrumented treadmill and escorted to a chair for three minutes of rest.

Biodex Balance System (BBS) Dynamic Limits of Stability (DLOS) (Biodex Medical Systems, Shirley, NY)

Participants were provided a verbal explanation and physical demonstration of the dynamic limits of stability (DLOS) test protocol. Participants were asked to step on the BBS stance platform for support rail and biofeedback display adjustment to ensure safety and comfort. Participant foot placement was adjusted on the BBS platform as standardized by BBS normative criteria based on participant height. One practice set, which consisted of three untimed practice trials, was performed prior to data collection trials. Participants stood on an unstable platform, shifted, and controlled their COG within their moving BOS in an attempt to direct a cursor on a biofeedback display from a centralized target to eight perimeter targets as quickly and with as little path deviation as possible. DLOS data were gathered over the course of three sets, each set consisted of three untimed trials with a three minute rest interval between each set (52). Balance platform stability was set at level eight of a 12 point scale with 12 being 
Robertson et al. Physical Therapy and Rehabilitation 2017,

most stable and one being least stable. An overall balance index was used for data analyses.

\section{Statistical analysis}

Pearson's product-moment correlation coefficient ( $r$ ) was calculated to describe the relationship between the TSTWT $\mathrm{EO}$ and the BBS DLOS overall index, measures of gait variance (COP, temporal, and spatial measures) primarily expressed as coefficient of variation (CV)(standard deviation/mean*100), primarily used to standardize unilateral data (with the exception of step width CV) and account for associated variability in the referenced data set, and percent difference, used to assess side to side symmetry, TSTWT EC, gait phase parameters (gait speed, cadence, double stance), and participant $\mathrm{BMI}$ and age. Linearity of data was determined from scatter diagram analysis. Multiple linear regression analyses were calculated to adjust for participant age. All analyses were performed using SPSS (version 21). Statistical significance was determined at an alpha value of less than $0.05(a<0.05)$.

\section{Results}

Forty-nine volunteers successfully completed the current test protocol. See Table 1 for participant characteristics.

Table 1. Participant Characteristics.

\begin{tabular}{ll}
\hline Age (years) & $40.1 \pm 17.1$ \\
Age Range (years) & $20-75$ \\
Body Mass Index $\left(\mathbf{k g} \bullet^{-2}\right)$ & $25.24 \pm 4.47$ \\
Participant Females (n) & 30 \\
Participant Males $(\mathbf{n})$ & 19 \\
\hline
\end{tabular}

Age and BMI are presented as mean \pm standard deviation

Pearson's product-moment correlation coefficient ( $r$ ) was performed between the TSTWT EO and research variables including the TSTWT EC, BBS DLOS Index, and the mFRQ demonstrating $p<0.05$ with a high positive correlation [53] demonstrated with the TSTWT EC ( $r=0.78)$, BBS DLOS overall index $(r=0.81)$, and a moderate positive correlation [53] with the $\mathrm{mFRQ}(r=0.61)$ (Table 2).

A moderate positive [53] relationship was demonstrated between the TSTWT EO and spatial measures of gait $(\mathrm{cm})$; step width percent difference $(r=0.61)$, step width CV $(r=0.64)$, and step length CV left and right ( $r=0.48$ and $r=0.49$, respectively). A moderate positive [53] relationship was demonstrated between the TSTWT EO and temporal measures of gait (s); step time CV ( $r=0.67)$ and swing time CV ( $r=0.57)$ (Table 2).

A low positive [53] to moderate positive [53]relationship was demonstrated between the TSTWT EO and COP measures $(\mathrm{mm})$; length of gait line left and right $(r=0.35$ and 0.31 , respectively), length of gait line $\mathrm{CV}(\mathrm{r}=0.52)$, single support line left and right $(r=0.51$ and $r=0.52$, respectively), single support line CV ( $r=0.48)$, and single support line percent dif- ference $(r=0.31 p=0.05)$. A moderate positive [53] relationship was demonstrated between the TSTWT EO and participant BMI $(r=0.50)$ and a moderate-high positive [53] relationship was demonstrated between the TSTWT EO and participant age $(r=0.69)$ (Table 2).

Multiple linear regression analyses were performed to adjust data for participant age. A high positive [53] relationship was demonstrated between the TSTWT EO and the BBS DLOS, TSTWT EC, step width CV, and step width percent difference $(r=0.86, r=0.84, r=0.81$, and $r=0.75$, respectively). A high positive [53] relationship was demonstrated between the TSTWT $E O$ and the $m F R Q(r=0.74), C O P$ single support line left and right $(r=0.73)$, COP length of gait line right $(r=0.73)$, double stance percentage $(r=0.72)$, step time variability $(r=0.71)$, single support line difference $(r=0.71)$, gait cadence $(r=0.71)$, step length difference $(r=0.70)$, and COP length of gait line left $(r=0.70)$ (Table 3).

\section{Discussion}

The TWT has historically been utilized as a reliable measure of dynamic walking balance in both clinical and research settings, yet to date, no studies have served to validate the TWT as a clinically efficacious measure of dynamic walking balance in a healthy population. The purpose of this investigation was to determine the concurrent validity of the ten step TWT eyes open (TSTWT EO) [8] as a tool for the assessment of dynamic walking balance in a cross-sectional healthy population between the ages of 20 and 75 years. The results from the current study supported the hypothesis that the TSTWT EO demonstrated concurrent validity as a test of dynamic walking balance in a cross-sectional, healthy population. The walk on floor eyes closed (WOFEC) version of the TWT had been validated, validity coefficient of .83, as a discriminatory test of walking dynamic balance in its ability to identify participants with labyrinthine defective ataxia [29], as a component of balance test batteries primarily utilized for elderly populations for the purpose of fall prediction [3-7], but had not been validated as an individualized measure of walking dynamic balance in a healthy population within the age range of 20 and 75 years. The TSTWT EO demonstrated moderate to high positive [53] correlation values with commonly used laboratory measures of dynamic balance (BBS DLOS, gait COP, time, and spatial measures, and the $\mathrm{mFRQ}$ ) in a healthy population between the ages of 20-75 years, with increased strength of association demonstrated when values were adjusted for participant age.

\section{COP parameters}

COP trajectory has been used to measure static and dynamic postural control and as a balance index for normal and pathological populations [11,13]. Bizovska et al. [11] demonstrated a difference in COP trajectory upon walking between healthy young and middle-aged women, alluding to age-associated reduced dynamic walking balance and the efficacy of gait COP 
Robertson et al. Physical Therapy and Rehabilitation 2017,

http://www.hoajonline.com/journals/pdf/2055-2386-4-12.pdf

Table 2. Pearson's Product Moment Correlation Coefficients between TSTWT EO, BBS DLOS, \& TSTWT EC and Measures of Dynamic Walking Balance.

\begin{tabular}{|c|c|c|c|}
\hline Research Variables & TSTWT EO & TSTWT EC & BBS DLOS \\
\hline Step Time $\mathrm{CV}^{\star}$ & $\begin{array}{l}\mathrm{r}=0.67 \\
\mathrm{p}=0.007\end{array}$ & $\begin{array}{l}\mathrm{r}=0.52 \\
\mathrm{p}=0.050\end{array}$ & $\begin{array}{l}\mathrm{r}=0.41 \\
\mathrm{p}=0.18\end{array}$ \\
\hline Swing Time $\mathrm{CV}^{\star}$ & $\begin{array}{l}\mathrm{r}=0.57 \\
\mathrm{p}=0.02\end{array}$ & $\begin{array}{l}-- \\
--\end{array}$ & -- \\
\hline Stride Time $\mathrm{CV}^{\star}$ & $\begin{array}{l}\mathrm{r}=0.43 \\
\mathrm{p}=0.04\end{array}$ & $\begin{array}{l}-- \\
--\end{array}$ & $\begin{array}{l}r=0.33 \\
p=0.03\end{array}$ \\
\hline Cadence (steps/min) & $\begin{array}{l}r=0.16 \\
p=0.33\end{array}$ & $\begin{array}{l}\mathrm{r}=0.09 \\
\mathrm{p}=0.58\end{array}$ & $\begin{array}{l}r=0.14 \\
p=0.37\end{array}$ \\
\hline Step Length Percent Difference & $\begin{array}{l}r=0.27 \\
p=0.31\end{array}$ & $\begin{array}{l}r=0.28 \\
p=0.08\end{array}$ & $\begin{array}{l}\mathrm{r}=0.18 \\
\mathrm{p}=0.26\end{array}$ \\
\hline Step Length $\mathrm{CV}^{\star}$ Left & $\begin{array}{l}r=0.48 \\
p=<0.05\end{array}$ & -- & $\begin{array}{l}-- \\
--\end{array}$ \\
\hline Step Length $\mathrm{CV}^{\star}$ Right & $\begin{array}{l}\mathrm{r}=0.49 \\
\mathrm{p}=<0.05\end{array}$ & -- & -- \\
\hline Step Width $(\mathrm{cm})$ & $\begin{array}{l}\mathrm{r}=0.61 \\
\mathrm{p}=0.006\end{array}$ & $\begin{array}{l}r=0.67 \\
p=0.03\end{array}$ & $\begin{array}{l}r=0.75 \\
p=0.01\end{array}$ \\
\hline Step Width $\mathrm{CV}^{*}$ & $\begin{array}{l}r=0.64 \\
p=0.006\end{array}$ & $\begin{array}{c}r=0.44 \\
p=0.02\end{array}$ & $\begin{array}{l}r=0.52 \\
p=0.008\end{array}$ \\
\hline COP Single Support Line Percent Difference (\%) & $\begin{array}{l}\mathrm{r}=0.31 \\
\mathrm{p}=0.05\end{array}$ & $\begin{array}{l}r=0.38 \\
p=0.01\end{array}$ & $\begin{array}{l}r=0.31 \\
p=0.05\end{array}$ \\
\hline COP Single Support Line Left (mm) & $\begin{array}{l}\mathrm{r}=0.51 \\
\mathrm{p}=0.001\end{array}$ & $\begin{array}{l}r=0.39 \\
p=0.01\end{array}$ & $\begin{array}{l}r=0.48 \\
p=0.002\end{array}$ \\
\hline COP Single Support Line Right (mm) & $\begin{array}{l}r=0.52 \\
p=0.0007\end{array}$ & $\begin{array}{l}r=0.37 \\
p=0.02\end{array}$ & $\begin{array}{l}r=0.53 \\
p=0.0005\end{array}$ \\
\hline COP Length of Gait Line Left (mm) & $\begin{array}{l}r=0.35 \\
p=0.03\end{array}$ & $\begin{array}{l}r=0.29 \\
p=0.07\end{array}$ & $\begin{array}{l}r=0.36 \\
p=0.024\end{array}$ \\
\hline COP Length of Gait Line Right (mm) & $\begin{array}{l}r=0.31 \\
p=0.05\end{array}$ & $\begin{array}{l}r=0.24 \\
p=0.14\end{array}$ & $\begin{array}{l}r=0.22 \\
p=0.17\end{array}$ \\
\hline Double Stance Percentage of Gait Cycle (\%) & $\begin{array}{l}\mathrm{r}=0.28 \\
\mathrm{p}=0.08\end{array}$ & $\begin{array}{l}r=0.18 \\
p=0.27\end{array}$ & $\begin{array}{l}r=0.27 \\
p=0.10\end{array}$ \\
\hline Gait Speed (m/s) & $\begin{array}{l}\mathrm{r}=0.24 \\
\mathrm{p}=0.13\end{array}$ & $\begin{array}{l}r=0.16 \\
p=0.32\end{array}$ & $\begin{array}{l}r=0.24 \\
p=0.13\end{array}$ \\
\hline $\begin{array}{l}\text { Biodex Balance System Dynamic Limits of Stability (stability } \\
\text { index) }\end{array}$ & $\begin{array}{l}\mathrm{r}=0.81 \\
\mathrm{p}=3.81 \mathrm{E}-12\end{array}$ & $\begin{array}{l}\mathrm{r}=0.73 \\
\mathrm{p}=0.02\end{array}$ & $\begin{array}{l}-- \\
--\end{array}$ \\
\hline Body Mass Index $(\mathrm{BMI})\left(\mathrm{kg} \cdot \mathrm{m}^{-2}\right)$ & $\begin{array}{l}r=0.50 \\
p=0.001\end{array}$ & $\begin{array}{l}\mathrm{r}=0.31 \\
\mathrm{p}=0.05\end{array}$ & $\begin{array}{l}r=0.50 \\
p=0.001\end{array}$ \\
\hline Modified Fall Risk Questionnaire & $\begin{array}{l}r=0.61 \\
p=7.1 \mathrm{E}-06\end{array}$ & -- & -- \\
\hline Age $(y)$ & $\begin{array}{l}r=0.69 \\
p=8.61 E-08\end{array}$ & $\begin{array}{l}\mathrm{r}=0.58 \\
\mathrm{p}=.000\end{array}$ & $\begin{array}{l}r=0.50 \\
p=0.000\end{array}$ \\
\hline Ten Step Tandem Walk Test Eyes Closed & $\begin{array}{l}\mathrm{r}=0.78 \\
\mathrm{p}=3.04 \mathrm{E}-10\end{array}$ & $\begin{array}{l}-- \\
--\end{array}$ & $\begin{array}{l}-- \\
--\end{array}$ \\
\hline
\end{tabular}

* Coefficient of variation(standard deviation $/$ mean $^{\star} 100$ )

trajectory parameters in classifying dynamic walking balance. Lee \& Chow [12] investigated COM and COP inclination angles, derived from both sagittal and frontal plane trajectories, in elderly walkers with a history of falls or unstable gait. Increased COM-COP frontal plane trajectory excursion identified elderly participants with dynamic walking balance abnormalities
[12]. COP measures reviewed in the present study were single support COP trajectory line, left and right, and length of COP trajectory gait line, left and right. A moderate correlation was demonstrated between theTSTWT EO and single support COP trajectory line, left and right $(r=0.51 p=0.001$ and $r=0.52$ $p=0.0007$, respectively). The strength of relationship increased 
Robertson et al. Physical Therapy and Rehabilitation 2017,

http://www.hoajonline.com/journals/pdf/2055-2386-4-12.pdf

Table 3. Pearson's Product Moment Correlation Coefficients Adjusted for Age.

\begin{tabular}{ll}
\hline Research Variables (Age Adjusted) & TSTWT EO \\
\hline Step Time CV & $\mathrm{r}=0.71 \mathrm{p}=0.000$ \\
Swing Time CV & $\mathrm{r}=0.70 \mathrm{p}=0.0001$ \\
Stride Time CV & $\mathrm{r}=0.70 \mathrm{p}=0.000$ \\
Cadence (steps/min) & $\mathrm{r}=0.71 \mathrm{p}=0.004$ \\
Step Length Percent Difference (\%) & $\mathrm{r}=0.70 \mathrm{p}=0.02$ \\
Step Width (cm) & $\mathrm{r}=0.75 \mathrm{p}=0.01$ \\
Step Width CV & $\mathrm{r}=0.81 \mathrm{p}=0.0001$ \\
COP Single Support Line Percent Difference (\%) & $\mathrm{r}=0.71 \mathrm{p}=0.01$ \\
COP Single Support Line Left (mm) & $\mathrm{r}=0.73 \mathrm{p}=0.03$ \\
COP Single Support Line Right (mm) & $\mathrm{r}=0.73 \mathrm{p}=0.03$ \\
COP Length of Gait Line Left (mm) & $\mathrm{r}=0.71 \mathrm{p}=0.02$ \\
COP Length of Gait Line Right (mm) & $\mathrm{r}=0.73 \mathrm{p}=0.006$ \\
Double Stance Percentage $(\%)$ & $\mathrm{r}=0.72 \mathrm{p}=0.04$ \\
Biodex Balance System Dynamic Limits of Stability (stability index) & $\mathrm{r}=0.86 \mathrm{p}=3.17 \mathrm{E}-08$ \\
Body Mass Index (BMI) (kg•m $\left.{ }^{-2}\right)$ & $\mathrm{r}=0.77 \mathrm{p}=0.0005$ \\
Modified Fall Risk Questionnaire (mFRQ) & $\mathrm{r}=0.74 \mathrm{p}=0.008$ \\
Ten Step Tandem Walk Test Eyes Closed & $\mathrm{r}=0.84 \mathrm{p}<0.000$ \\
\hline
\end{tabular}

${ }^{\star}$ Coefficient of variation

to a high-positive [53] correlation when adjusted for age $(r$ $=0.73 \mathrm{p}=0.03$ left and right). A low-positive [53] correlation was determined between the TSTWT EO and length of COP trajectory gait line, left and right $(r=0.35 p=0.03$ and $r=0.31$ $p=0.05$, respectively) yet the correlational strength increased to a high-positive [53] relationship when data were adjusted for age ( $r=0.70 p=0.02$ left and $r=0.73 p=0.006$ right). This low to high positive relationship between COP trajectory measures and the TSTWT EO implied that the relationship between dynamic walking balance and the TSTWT EO was strong when age was accounted for and low to moderate when age was not considered.

\section{Temporal parameters}

Irregular temporal parameters of gait have been associated with aging, falls, and a variety of diseases [18]. Step time variability has been associated gait pattern changes identified with aging, traumatic brain injury, diabetes mellitus associated peripheral neuropathy, Parkinson's disease, Alzheimer's disease, affective psychiatric disorder, fall risk, and falls [18]. Richardson et al. [18] studied the relationship between step length and step time variability on both smooth and irregular walking surfaces in older participants with neuropathy. An inverse relationship was reported between step length and step time variability, which indicated that as step length decreased, as found with reduced gait stabilization, step time variability increased linking increased step time variability with reduced walking stability, hence, reduced walking balance [18]. The TSTWT EO demonstrated moderate [53] $(r=0.67$ $\mathrm{p}=0.007)$ and high-positive [53] $(\mathrm{r}=0.71 \mathrm{p}=0.000)$ correlations with step time $\mathrm{CV}$ relative to unadjusted and data adjusted for participant age, respectively. Frenkel-Toledo et al. [16] found an independent relationship between gait stability and swing-time variability, hypothesized as a valid indicator of gait steadiness and fall risk, indicative of dynamic walking balance. A moderate-high positive[53] relationship, without and with age adjustment $(r=0.57 p=0.02, r=0.71 p=0.000$, respectively) was found between swing-time CV and the TSTWT EO, indicating a moderate to high positive [53] relationship between dynamic walking balance and the TSTWT EO. Gait stride-to-stride fluctuations have been associated with falls, individuals at risk for falls, and normal aging $[14,15]$. Community-dwelling older adults demonstrated a fivefold increase in the possibility of falls upon only a moderate increase in stride time variability, indicative of gait instability and walking dynamic balance $[14,15]$. The TSTWT EO demonstrated a moderate [53] $(r=0.43 p=0.04)$ correlation with stride time CV and a high positive [53] $(r=0.70 p=0.000)$ correlation when adjusted for participant age, demonstrating a strong association between the TSTWT EO and dynamic walking balance.

\section{Spatial parameters}

Stride to stride variability has been associated with risk of disability [19] and falls $[20]$. Brach et al. $[19,20]$ found that both excessive and reduced step width variability were indicative of gait instability as the ability to modify step width with gait was shown as a compensatory mechanism used to stabilize the COM under varying gait conditions, such as altered speed 
and/or uneven terrain. An age-associated increase in step width variability allowed for accurate participant age-range identification, which associated step width variability with risk for future falls $[15,22]$. The TSTWT EO demonstrated a high positive [53] correlation with step width with and without age adjustment $(r=0.75 p=0.01$ and $r=0.61 p=0.006$, respectively). A high positive correlation existed between the TSTWT EO and step width CV when data was adjusted for age $(r=0.81$ $p=0.000$ ), which suggested a strong association between the TSTWT EO and dynamic walking balance.

\section{Biodex Balance System Dynamic Limits of Stability}

The TSTWT EO demonstrated a high positive [53] correlation with the BBS DLOS test both prior to and with age-adjusted participant data $(r=0.81 p=0.000$ and $r=0.86 p=0.000$, respectively). Aydog et al. [23] compared dynamic balance between two groups of participants, healthy controls and participants with lower extremity involved rheumatoid arthritis (RA) and found dynamic balance was negatively affected in individuals with RA compared to healthy control participants which was objectively demonstrated using the BBS DLOS [23]. Mohan et al. utilized the BBS DLOS system to quantify dynamic balance in individuals with spinocerebellar ataxia in order to determine the efficacy of rehabilitative interventions [25]. Dynamic balance was compared between participants with diagnosed spinocerebellar ataxia and age matched healthy controls [25]. The BBS DLOS was reported to be the best predictor of pathological dynamic balance [25]. Pickerill \& Harter attempted to establish the concurrent validity of two DLOS balance systems, BBS and the NeuroCom Smart Balance Master (Clackamas, OR) and found a low positive, yet non-significant correlation $(r=0.33 p=0.12)$ between the two systems for overall directional balance control measures [1]. The non-significant low correlation was attributed to differences in testing protocols [1]. The BBS DLOS provided a greater challenge to participant's functional stability due to its unstable standing base and was reported to uniquely challenge dynamic balance, yet Pickerill and Harter [1] found the NeuroCom (Clackamas, OR) to be more reliable as a LOS measure. Pickerill \& Harter [1] reported the NeuroCom LOS maintained a fixed BOS, the BBS DLOS maintained foot position, yet the unstable stance platform of the BBS DLOS system continuously altered participant's BOS resulting in a greater dynamic balance challenge. Hinman [27] found the BBS to be a reliable instrument for use in testing and quantifying dynamic balance in a healthy population. Limits of stability testing using the BBS was more difficult for participants than LOS testing using the NeuroCom due to the BBS's unstable stance platform [27]. Hinman [27] suggested that dynamic balance testing using the BBS LOS test may have been too difficult for a participant population with pre-existing balance impairments as a percentage of test participants were unable to complete the DLOS tests without using upper extremity assistance to prevent complete loss of balance. Schmitz \&
Arnold [26] determined the intertester and intratester reliability values for the BBS DLOS for dynamic balance through a unilateral stance protocol based on anterior to posterior $(A-P)$, medial lateral $(M-L)$, and overall stability indices. Intertester and intra-tester ICC stability scores ranged from .70 to .42 and .80 to .43 , respectively [26]. Schmitz \& Arnold [26] concluded that the BBS was a reliable tool for testing dynamic stability. The BBS DLOS system appeared to accurately and uniquely depict dynamic balance in both pathological and healthy populations. The strong association found between the TSTWT EO and BBS DLOS suggested a strong relationship between dynamic balance and the TSTWT EO. Despite methodological controls built into this research design, a degree of subjectivity in regards to TSTWT LOB assignment may have influenced test results. Not with standing the reported clinical efficacy of the TSTWT EO as reported in the current study, careful consideration regarding clinical application of this study's findings should be applied to dynamic walking balance test selection as the participant population was limited to a healthy population and did not include a clinical population. The propensity of the TSTWT EO to maximally narrow an individual's BOS may overly challenge a clinical population with diminished dynamic walking capacity. The purpose of this study was to validate the TSTWT's capacity to assess dynamic walking balance in a healthy population, not a clinically balance impaired population. A future direction for this research should entail usage of similar test methodology in a participant population with known diminished dynamic walking capacity.

\section{Conclusion}

There appeared to be a moderate to high positive [53] relationship between theTSTWT EO and commonly used laboratory measures of dynamic balance (BBS DLOS and gait COP, temporal, and spatial parameters) in a healthy population between the ages of 20-75 years, with increased strength of association demonstrated when values were adjusted for participant age. The TSTWT EO did not demonstrate criterion validity as a measure of walking dynamic balance since no criterion standard regarding dynamic walking balance has been identified, yet, due to the strength of association between the TSTWT EO and commonly used measures of dynamic walking balance, the TSTWT EO demonstrated convergent-construct validity as a measure of walking dynamic balance. The TWT has historically been utilized as a measure of dynamic walking balance due to its propensity to maximally narrow the base of support of the tested individual [2], but theTSTWT EO was never validated as a test of dynamic walking balance in a healthy population between the ages of 20 and 75 years. The TSTWT EO was found to be a clinically efficacious quantitative and qualitative measure of dynamic walking balance which demonstrated significant clinical utility compared with the afore-mentioned laboratory tests utilized as tests of dynamic walking balance considering cost and portability. 
Robertson et al. Physical Therapy and Rehabilitation 2017,

http://www.hoajonline.com/journals/pdf/2055-2386-4-12.pdf

doi: 10.7243/2055-2386-4-12

\section{List of abbreviations}

BBS: Biodex Balance System

BMI: Body mass index

BOS: Base of support

$\mathrm{cm}$ : centimeters

COM: Center of mass

COP: Center of pressure

$\mathrm{CV}$ : Coefficient of variance

DLOS: Dynamic limits of stability

LOS: Limits of stability

mFRQ: Modified Fall Risk Questionnaire

$\mathrm{mm}$ : millimeters

s: seconds

TWT: Tandem walk test

TSTWT EC: Ten step tandem walk test eyes closed

TSTWT EO: Ten step tandem walk test eyes open

USA: United States of America

\section{Competing interests}

The authors declare that they have no competing interests.

Authors' contributions

\begin{tabular}{|l|c|c|}
\hline Authors' contributions & MR & RG \\
\hline Research concept and design & $\checkmark$ & $\checkmark$ \\
\hline Collection and/or assembly of data & $\checkmark$ & -- \\
\hline Data analysis and interpretation & $\checkmark$ & -- \\
\hline Writing the article & $\checkmark$ & -- \\
\hline Critical revision of the article & $\checkmark$ & -- \\
\hline Final approval of article & $\checkmark$ & $\checkmark$ \\
\hline Statistical analysis & $\checkmark$ & -- \\
\hline
\end{tabular}

\section{Acknowledgements}

The authors would like to thank Dr. Robert Axtell for his advisory and editorial contribution to this project.

Publication history

Editor: Catherine Ortega, University of Texas Health Science Center, USA.

Received: 29-Jun-2017 Final Revised: 01-Sep-2017

Accepted: 30-Sep-2017 Published: 15-Oct-2017

\section{References}

1. Pickerill ML and Harter RA. Validity and reliability of limits-of-stability testing: a comparison of 2 postural stability evaluation devices. J Athl Train. 2011; 46:600-6. | PubMed Abstract | PubMed FullText

2. Lark SD and Pasupuleti S. Validity of a functional dynamic walking test for the elderly. Arch Phys Med Rehabil. 2009; 90:470-4. | Article | PubMed

3. Rose DJ, Lucchese $\mathrm{N}$ and Wiersma LD. Development of a multidimensional balance scale for use with functionally independent older adults. Arch Phys Med Rehabil. 2006; 87:1478-85. | Article | PubMed

4. Hernandez D and Rose DJ. Predicting which older adults will or will not fall using the Fullerton Advanced Balance scale. Arch Phys Med Rehabil. 2008; 89:2309-15. | Article | PubMed

5. Clemson L, Singh MF, Bundy A, Cumming RG, Weissel E, Munro J, Manollaras K and Black D. LiFE Pilot Study: A randomised trial of balance and strength training embedded in daily life activity to reduce falls in older adults. Aust Occup Ther J. 2010; 57:42-50. | Article | PubMed

6. Hiura $M$, Nemoto $H$, Nishisaka $K$, Higashi $K$ and Katoh $T$. The association between walking ability and falls in elderly Japanese living in the community using a path analysis. J Community Health. 2012; 37:957-62. | Article | PubMed

7. Dargent-Molina P, Douchin MN, Cormier C, Meunier PJ and Breart G. Use of clinical risk factors in elderly women with low bone mineral density to identify women at higher risk of hip fracture: The EPIDOS prospective study. Osteoporos Int. 2002; 13:593-9. | Article | PubMed

8. Robertson M. Comparison of dynamic walking balance in healthy participants $\mathbf{2 0 - 2 9}$ and $\mathbf{7 0 - 7 9}$ years utilizing the tandem walk test. 2016. | Website

9. Shimada H, Obuchi S, Kamide N, Shiba Y, Okamoto M and Kakurai S. Relationship with dynamic balance function during standing and walking. Am J Phys Med Rehabil. 2003; 82:511-6. | Article | PubMed

10. Vereeck L, Wuyts F, Truijen S and Van de Heyning P. Clinical assessment of balance: normative data, and gender and age effects. Int J Audiol. 2008; 47:67-75. | Article | PubMed

11. Bizovska L, Svoboda Z, Kutilek P, Janura M, Gaba A and Kovacikova Z. Variability of centre of pressure movement during gait in young and middle-aged women. Gait Posture. 2014; 40:399-402. | Article | PubMed

12. Lee HJ and Chou LS. Detection of gait instability using the center of mass and center of pressure inclination angles. Arch Phys Med Rehabil. 2006; 87:569-75. | Article | PubMed

13. Jamshidi N, Rostami M, Najarian S, Menhaj MB, Saadatnia M and Salami F. Differences in center of pressure trajectory between normal and steppage gait. J Res Med Sci. 2010; 15:33-40. | PubMed Abstract | PubMed FullText

14. Hausdorff JM. Gait variability: methods, modeling and meaning. $J$ Neuroeng Rehabil. 2005; 2:19. | Article | PubMed Abstract | PubMed FullText

15. Hausdorff JM, Rios DA and Edelberg HK. Gait variability and fall risk in community-living older adults: a 1-year prospective study. Arch Phys Med Rehabil. 2001; 82:1050-6. | Article | PubMed

16. Frenkel-Toledo S, Giladi N, Peretz C, Herman T, Gruendlinger L and Hausdorff JM. Effect of gait speed on gait rhythmicity in Parkinson's disease: variability of stride time and swing time respond differently. J Neuroeng Rehabil. 2005; 2:23. | Article | PubMed Abstract | PubMed FullText

17. Beauchet O, Allali G, Annweiler C, Bridenbaugh S, Assal F, Kressig RW and Herrmann FR. Gait variability among healthy adults: low and high stride-to-stride variability are both a reflection of gait stability. Gerontology. 2009; 55:702-6. | Article | PubMed

18. Richardson JK, Thies SB, DeMott TK and Ashton-Miller JA. A comparison of gait characteristics between older women with and without peripheral neuropathy in standard and challenging environments. J Am Geriatr Soc. 2004; 52:1532-7. | Article | PubMed

19. Brach JS, Berthold R, Craik R, VanSwearingen JM and Newman AB. Gait variability in community-dwelling older adults. J Am Geriatr Soc. 2001; 49:1646-50. | Article | PubMed

20. Brach JS, Berlin JE, VanSwearingen JM, Newman AB and Studenski SA. Too much or too little step width variability is associated with a fall history in older persons who walk at or near normal gait speed. $J$ Neuroeng Rehabil. 2005; 2:21. | Article | PubMed Abstract | PubMed FullText

21. Grabiner MD and Troy KL. Attention demanding tasks during treadmill walking reduce step width variability in young adults. J Neuroeng Rehabil. 2005; 2:25. | Article | PubMed Abstract | PubMed FullText

22. Owings TM and Grabiner MD. Variability of step kinematics in young and older adults. Gait Posture. 2004; 20:26-9. | Article | PubMed

23. Aydog E, Bal A, Aydog ST and Cakci A. Evaluation of dynamic postural balance using the Biodex Stability System in rheumatoid arthritis patients. Clin Rheumatol. 2006; 25:462-7. | Article | PubMed

24. Sieri T and Beretta G. Fall risk assessment in very old males and females living in nursing homes. Disabil Rehabil. 2004; 26:718-23. | Article | PubMed

25. Mohan G, Pal PK, Sendhil KR, Thennarasu K and Usha BR. Quantitative evaluation of balance in patients with spinocerebellar ataxia type 1: a 
Robertson et al. Physical Therapy and Rehabilitation 2017,

case control study. Parkinsonism Relat Disord. 2009; 15:435-9. | Article I PubMed

26. Schmitz $R$ and Arnold B. Intertester and intratester reliability of a dynamic balance protocol using the Biodex Stability System. J Sport Rehabil. 1998; 7:95-101. | Article

27. Hinman MR. Factors affecting reliability of the Biodex Balance System: a summary of four studies. J Sport Rehabil. 2000; 9:240-252. | Article

28. Graybiel A and Fregly AR. A New Quantitative Ataxia Test Battery. Nsam919. Res Rep U S Nav Sch Aviat Med. 1965; 15:1-38. | PubMed

29. Fregly AR, Graybiel A and Smith MJ. Walk on floor eyes closed (WOFEC): a new addition to an ataxia test battery (No. NAMRL-1144). NAVAL AEROSPACE MEDICAL RESEARCH LAB PENSACOLA FL. 1971; 1-12. | Pdf

30. Graybiel A, Smith CR, Guedry FE, Jr., Miller EF, Fregly AR and Cramer DB. Idiopathic progressive vestibular degeneration. Ann Otol Rhinol Laryngol. 1972; 81:165-78. | Article | PubMed

31. Speers RA, Ashton-Miller JA, Schultz AB and Alexander NB. Age differences in abilities to perform tandem stand and walk tasks of graded difficulty. Gait Posture. 1998; 7:207-213. | Article | PubMed

32. Nelson ME, Layne JE, Bernstein MJ, Nuernberger A, Castaneda C, Kaliton D, Hausdorff J, Judge JO, Buchner DM, Roubenoff R and Fiatarone Singh MA. The effects of multidimensional home-based exercise on functional performance in elderly people. J Gerontol A Biol Sci Med Sci. 2004; 59:154-60. | PubMed

33. Nelson ME, Fiatarone MA, Morganti CM, Trice I, Greenberg RA and Evans WJ. Effects of high-intensity strength training on multiple risk factors for osteoporotic fractures. A randomized controlled trial. JAMA. 1994; 272:1909-14. | Article | PubMed

34. Chu LW, Chi I and Chiu AY. Incidence and predictors of falls in the chinese elderly. Ann Acad Med Singapore. 2005; 34:60-72. | Pdf I PubMed

35. Cho BL, Scarpace D and Alexander NB. Tests of stepping as indicators of mobility, balance, and fall risk in balance-impaired older adults. J Am Geriatr Soc. 2004; 52:1168-73. | Article | PubMed

36. Morris R, Harwood RH, Baker R, Sahota O, Armstrong S and Masud T. A comparison of different balance tests in the prediction of falls in older women with vertebral fractures: a cohort study. Age Ageing. 2007; 36:78-83. | Article | PubMed

37. Tinetti ME, Speechley $M$ and Ginter SF. Risk factors for falls among elderly persons living in the community. N Engl J Med. 1988; 319:17017. | Article | PubMed

38. Dargent-Molina P, Favier F, Grandjean H, Baudoin C, Schott AM, Hausherr $E$, Meunier PJ and Breart G. Fall-related factors and risk of hip fracture: the EPIDOS prospective study. Lancet. 1996; 348:145-9. | Article | PubMed

39. Schrager MA, Kelly VE, Price R, Ferrucci L and Shumway-Cook A. The effects of age on medio-lateral stability during normal and narrow base walking. Gait Posture. 2008; 28:466-71. | Article | PubMed Abstract | PubMed FullText

40. Howe JA, Inness EL, Venturini A, Williams JI and Verrier MC. The Community Balance and Mobility Scale--a balance measure for individuals with traumatic brain injury. Clin Rehabil. 2006; 20:885-95. | Article | PubMed

41. Cohen HS, Mulavara AP, Peters BT, Sangi-Haghpeykar H and Bloomberg JJ. Tests of walking balance for screening vestibular disorders. J Vestib Res. 2012; 22:95-104. | Article | PubMed Abstract | PubMed FullText

42. Celebisoy N, Bayam E, Gulec F, Kose T and Akyurekli O. Balance in posterior and horizontal canal type benign paroxysmal positional vertigo before and after canalith repositioning maneuvers. Gait Posture. 2009; 29:520-3. | Article | PubMed

43. Oluwole OS, Onabolu AO, Link $\mathrm{H}$ and Rosling $\mathrm{H}$. Persistence of tropical ataxic neuropathy in a Nigerian community. J Neurol Neurosurg Psychiatry. 2000; 69:96-101. | Article | PubMed Abstract | PubMed FullText

44. Cohen HS, Mulavara AP, Peters BT, Sangi-Haghpeykar H, Kung DH, Mosier $\mathrm{DR}$ and Bloomberg JJ. Sharpening the tandem walking test for screening peripheral neuropathy. South Med J. 2013; 106:565-9. | Article | PubMed Abstract | PubMed FullText
45. Torvinen S, Kannu P, Sievanen $H$, Jarvinen TA, Pasanen M, Kontulainen S, Jarvinen TL, Jarvinen M, Oja P and Vuori I. Effect of a vibration exposure on muscular performance and body balance. Randomized cross-over study. Clin Physiol Funct Imaging. 2002; 22:145-52. | Article | PubMed

46. Berkman LF, Seeman TE, Albert M, Blazer D, Kahn R, Mohs R, Finch C, Schneider E, Cotman C, McClearn G and et al. High, usual and impaired functioning in community-dwelling older men and women: findings from the MacArthur Foundation Research Network on Successful Aging. J Clin Epidemiol. 1993; 46:1129-40. | Article | PubMed

47. Gill J, Allum JH, Carpenter MG, Held-Ziolkowska M, Adkin AL, Honegger $\mathrm{F}$ and Pierchala K. Trunk sway measures of postural stability during clinical balance tests: effects of age. J Gerontol A Biol Sci Med Sci. 2001; 56:M438-47. | PubMed

48. Allum JH, Adkin AL, Carpenter MG, Held-Ziolkowska M, Honegger F and Pierchala K. Trunk sway measures of postural stability during clinical balance tests: effects of a unilateral vestibular deficit. Gait Posture. 2001; 14:227-37. | Article | PubMed

49. Tomchuk D. Companion guide to measurement and evaluation for kinesiology. Jones \& Bartlett Publishers: Sudbury, MA. 2011; 84.

50. Clary S, Barnes C, Bemben D, Knehans A and Bemben M. Effects of ballates, step aerobics, and walking on balance in women aged 50-75 years. J Sports Sci Med. 2006; 5:390-9. | PubMed Abstract | PubMed FullText

51. Rubenstein LZ, Vivrette R, Harker JO, Stevens JA and Kramer BJ. Validating an evidence-based, self-rated fall risk questionnaire (FRQ) for older adults. J Safety Res. 2011; 42:493-9. | Article | PubMed

52. Clark S, Rose DJ and Fujimoto K. Generalizability of the limits of stability test in the evaluation of dynamic balance among older adults. Arch Phys Med Rehabil. 1997; 78:1078-84. | Pdf | PubMed

53. Hinkle DE, Wiersma $W$ and Jurs SG. Rule of Thumb for Interpreting the Size of a Correlation Coefficient. Derived from $5^{\text {th }}$ ed., Applied Statistics for the Behavioral Sciences ( $5^{\text {th }}$ ed.). 2003. | Pdf

\section{Citation:}

Robertson M and Gregory R. Concurrent validation of the tandem walk test as a measure of dynamic walking balance in a healthy population. Phys Ther Rehabil. 2017; 4:12.

http://dx.doi.org/10.7243/2055-2386-4-12 